\title{
The Effect of CEO's Strategic Leadership on Organizational Satisfaction through Communication: Focusing on the Moderating Effect of Management Trust Groups
}

\author{
*Nam-Sik Yun , Doctoral Student, Dept. of Smart Convergence Consulting, Han sung University, Seoul, \\ 02879, Korea, muntiger79@hansung.ac.kr \\ Seok Kee Lee, Professor, Dept. of Computer Engineering, Han sung University, Seoul, 02879, Korea, \\ seelee@hansung.ac.kr \\ *Corresponding Author
}

\begin{abstract}
The purpose of this study is to investigate the effects of CEO's strategic leadership on organizational communication and job satisfaction and the moderating effects of trust in $\mathrm{m}$ anagement.

The subjects of this study were 276 small and medium-sized enterprises in Gyeonggi-do, and the mediating and moderating effects were analyzed by using a structural equation model.

The results of this study are as follows: First, strategic leadership has a significant effect on communication. Second, communication had a significant effect on job satisfaction, and third, strategic leadership had a positive effect on job satisfaction. Fourth, it was confirmed that the high-level group with high management trust had a high moderating effect on the ef fect of strategic leadership on communication ability and job satisfaction.

The results of this study suggest that when CEOs face a multi-variable environment today, th ey continue their efforts to have strategic leadership, they can expect the management perfor mance according to the secondary organizational performance by improving the management $p$ erformance as well as smooth communication among the employees and work efficiency accor ding to job satisfaction.
\end{abstract}

Keywords: Strategic leadership, communication, job satisfaction, trust in management $\begin{array}{lll}\text { Received: 08.12.2020 } & \text { Accepted: 10.01.2021 } & \text { Published: 06.02.2021 }\end{array}$

\section{INTRODUCTION}

Modern corporate organizations face a very uncertain business environment, and with this uncertain business environment, the maintenance and development of the company can never be optimistic. The modern business management environment is an age of infinite competition without borders. In the late 20th century, the chronic and structural problems of our economy, high cost and low efficiency, have even made the IMF a ruinous situation. However, there are companies that continue to grow and develop even in the midst of recession and recession. In this fact, it can be interpreted that the influence of the management environment is not the cyclical dimension of economic fluctuations as in the past, but the era is now determined in terms of competitiveness of the organization. In other words, in the age of infinite competition, organizational rise and fall are not determined only by external environments, but by the power of the organization to respond to these environments, that is, the ability, qualities, and motivation of the organization members.

In addition, in order to achieve the goals pursued by the organization efficiently, organic cooperation between individual members of the organization and the group consisting of these individuals is necessary. One of the essential organizational factors that induce, coordinate these organic systems and connect them to organizational performance is leadership (Yoon Se-ra, 2017)[1].

Leadership develops cooperative culture within the organization, forms smooth communication within the organization, ultimately creates an environment in which organizational goals and individual values are matched to create an environment in which organizational commitment can be immersed in the organization, and organizational commitment can soon create high results and lead to job satisfaction (Lee Hyun-joo, 2017)[2]. Smooth communication within an organization means the process of sharing opinions with each other and delivering various information generated within the organization. When the organization is an organism that interacts to achieve common goals by gathering more than two people, 
this interaction is promoted because of smooth communication, and therefore, it is important in that interpersonal relationship and management function cannot work without communication (Kim Janghwan, 2012)[3]. This smooth communication also resolves the gap between the members and coordinates and integrates these activities. When such smooth communication settles in an organization, the functions within the organization can be integrated to maintain and develop the system, ultimately achieving organizational goals, and motivating members to strengthen job satisfaction (Song Ki-hyun, 2013)[4].

Therefore, it is expected that CEO's leadership will have a positive effect on communication and job satisfaction in the organization, and smooth communication can lead to high job satisfaction.

Especially, when CEO has strategic leadership that is a combination of absorption ability, which means learning ability, adaptability ability, which is ability to change, and management ability that combines discernment and appropriate timing, communication and job satisfaction in organization can be further improved. Kang Hyun (2017) suggested that strategic human resource management through strategic leadership can be linked to job satisfaction by favoring talented people, providing autonomy of work, and promoting smooth communication between organizations[5].

Marshall (2000), on the other hand, emphasized the importance of trust while promoting the quality of cooperation that induces the spontaneity and job satisfaction of members who promote communication[6] .In other words, it is possible to promote activeness and interaction among members who are trust-enriched organizations, so that they can expect more understanding, cooperation, commitment and commitment from members, so they have the potential to go to an organization with high performance. Therefore, it is suggested that the trust of management created by the strategic leadership of CEOs can further promote communication and job satisfaction within the organization.

According to the previous studies on the influence of strategic leadership on organization, Yoo Jung-ok \& Choi Sung-woo (2014) explored the capacity of strategic leadership of elementary and secondary school principals[7]. Kim Young-chun \& Baek Yoo-sung (2011) derived the components of strategic leadership centered on high-level military service[8]. Yoon So-hee (2020) analyzes the educational needs for strengthening the strategic leadership capacity of the principal, and the analysis of strategic leadership is limited to the military and education fields[9]. Although Kim Sung-geon(2013) is verifying the impact of M\&A CEO's strategic leadership on corporate performance[10], There is a lack of research to verify exactly which members' attributes lead to performance. Therefore, this study aims to verify the influence of CEO's strategic leadership on communication and job satisfaction in the organization based on the background of this study and limitations of previous studies.

\section{Theoretical background and prior research}

\subsection{CEO's Strategic Leadership}

Strategic leadership was established as a theory as the study on strategic leaders and strategic leadership was actively developed as a concept that emerged from the Upper Echelon Theory that the personal characteristics of CEOs can affect corporate strategy and performance. Finkelstein \& Hambrick (1996) defined strategic leadership as a total influence of stakeholders such as top executives belonging to the upper levels of the corporate organization on the structure and future of the organization, and discussed the influence of the management on the organizational performance of the individual characteristics and the work process method[11]. In the course of promoting the change for organizational development, the research of Ireland \& Hitt(1999) defined the essential future prediction, vision presentation, strategic thinking, and collaboration ability with others as strategic leadership[12]. They analyzed the components of strategic leadership and achievement strategies. Rowe (2001) defined strategic leadership as leadership that affects the members to voluntarily implement and handle everyday decisions in order to secure long-term survival and short-term financial stability of the organization [13]. On the other hand, Yoon So-hee(2020)'s study summarizes the characteristics of strategic leadership by strategic decision making for future orientation and change, and emphasizes that unlike existing leaders who rely on clear information, strategic leaders pay attention to various phenomena inside and outside the organization and make decisions based on uncertain information[9]. Finally, Hambrick \& Mason(1984) presented the attributes of strategic leadership by consisting of goal/vision presentation, strategic control core competencies, and human resource development[14]. In conclusion, strategic leadership is a total influence to promote organizational performance by strategically controlling and developing human resources to present goals and visions for change to members by predicting the future according to strategic thinking based on information with high uncertainty and to achieve this. 


\subsection{Communication}

Communication has the meaning of common and sharing; it is called communication in English, and includes the same meaning as sharing with others[15]. Etymologically, communication is an act of sharing or sharing people's information, opinions, emotions, attitudes, facts, and thoughts, and conveys emotions and meanings through exchange messages with humans.

Communication also plays an important role in communicating with people who are indispensable to living with others and includes all means of exchanging influence through mutual conversation[16].

Communication skills are important enough to determine whether leaders of organizations succeed in improving organizational structure (Beach \& Reinhartz, 2000)[17]. Therefore, in order to form relationships, build trust, and respect each other through communication, effective organizational structure improvement should be secured (Green, 2001)[18], clear and smooth communication is essential for organizational members. (According to Hamilton, C., Parker, 1993), communication is a process of sharing people's thoughts, thoughts, and emotions, defined in a way that we understand together[19]. Communication can be used to exchange information and data with organizational members, and to promote relationships to make decisions (Reinhartz \& Beach, 2004)[20]. Son Jeong-hoon(2003) studied the effect of communication ability on job satisfaction and the higher communication ability, the higher job satisfaction. Based on this, the definition of communication is a total act of exchanging more than two people's intentions, emotions, and information, and is an interaction process through language and nonverbal means [21].

In this study, communication was defined as a process of information transfer among organizational members.

\subsection{Job satisfaction}

In order to examine the concept of job satisfaction, the definition of meaning in the dictionary is 'satisfaction with the job', which is somewhat different among scholars. Job satisfaction is generally considered an individual's attitude, but sometimes also used as a general level of attitude within the group. Job satisfaction is also used as a concept of overall satisfaction and applies to some individual jobs. First, Hoppock (1935) argued that job satisfaction is a state that integrates all of the physiological, physiological, and environmental situations of the organizational members [22]. Reitz (1981) is defined as three elements: behavior, information, and emotion, as emotional responses to an individual's emotions or duties, and as a specific concept of job satisfaction given that it is fundamentally done by various functions of the individual as well as various strengths [23]. Locke (1976) viewed job satisfaction as an interaction between 'personal value' and 'awareness of work and environment', based on the approach that everyone is in a defined hierarchy of the same desire and opposes the logic that human needs vary from person to person [24]. When defining the concept of job satisfaction based on the definition of scholars, job satisfaction refers to a favorable attitude toward individual job, and the group with high job satisfaction showed a positive attitude toward job, but the group with low job satisfaction showed a negative attitude toward job.

Therefore, job satisfaction is an element that affects the desirable behavior of the organizational members, has a significant effect on the organizational performance, and gives stability to the individual, so it is important as an organization or member. In this study, positive response to the factors related to the organizational members is defined as job satisfaction.

\subsection{Management trust}

According to Cook and Wall (1980), trust is the trust in the words and actions of others and is defined as the trust in the ability to perform according to the good will of others[25]. Mayer, Davis and Scorman (1995) predicted that the other person would do something important to him and said he would be willing to accept the vulnerability that could occur in response to the other person's actions[26]. Lee Young-seok (2004) argued that trust in leaders is a psychological state that is willing to accept the risk burden that can be applied to them based on positive expectations of leaders' actions or intentions. In conclusion, this study defined trust as the level of willingness to accept uncertain situations based on the relationship between organizational members and to act optimistically based on expectations and beliefs[27].

The existing organizational theory and management theory organization are interested in rational and reasonable appearance and activities, and organizational trust implies that individual emotion and mood are considered part of organizational theory (Fineman, 1993)[28]. The quality of life of human beings is emerging as an important value, and the emotional state of individuals affects the performance of the organization, so reliability can be seen to have a great influence.

Kim Myung-eon(2000) mentioned that the members of the organization feel trust in the manager who 
builds the community relationship considering the situation, and that the emotional dimension of consideration and good will has a great influence on trust[29]. Lee Ju-il(2001) collected trust and distrust and showed the highest rate of the boss's caring behavior, which showed that the manager's interest and caring behavior had the most important correlation with the manager's trust, and claimed that it was the most important[30].

In this study, we defined management trust as a willingness to accept uncertain situations and act optimistically and optimistically.

\subsection{A Study on the Relationship among CEO's Strategic Leadership, Communication in Organization, and Job Satisfaction}

Shin Seung-in \& Choi Eun-soo (2018) analyzed the structural relationship between the moderate rationalist leadership of the secondary school principal and the level of communication within the organization, learning organization culture, teacher's teaching ability and activation of the teacher learning community. As a result, the study verified that the warm rationalist leadership had a direct positive effect on communication within the organization [31]. In addition, Gong Man-seok(2018) verified the relationship between the empowering leadership, directive leadership, safety communication and safety behavior of air force combat pilots with the controlled mediating effect of safety motivation. As a result of the study, it was confirmed that the safety motivation had a significant moderating effect on the impact of empowering leadership on safety communication [32].These results suggest that various different attributes of leadership have a significant correlation with communication ability, and therefore, strategic leadership that this study focuses on can promote smooth communication of organizations.

Meanwhile, Kang Se-hyun \& Lee Yoon-cheol(2019) analyzed the effects of transformational leadership and authentic leadership of airline cabin team leader on job satisfaction and organizational commitment, focusing on the mediating effects of self-efficacy and LMX. The results of this study showed that both transformational and authentic leadership of cabin team leaders significantly promoted job satisfaction and organizational commitment of flight attendants[33]. In addition, Lee Kang-kook \& Lee Sun-gyu (2015) verified the moderating effect of active participation of subordinates on the effect of commander's transformational and transactional leadership on job satisfaction. As a result of the study, it was confirmed that active participation of subordinates had a significant moderating effect on the effect of transformational leadership on job satisfaction [34].In conclusion, it is suggested that various leadership attributes can have positive (+) correlation with job satisfaction, and therefore, strategic leadership is expected to have a positive effect on job satisfaction of the organization members.

Finally, the study on the effect of communication in an organization on job satisfaction is as follows. Lim Seung-ju(2016) identified the relationship among self-esteem, communication ability, and job satisfaction of local government health officials, and it was confirmed that communication ability and job satisfaction had a positive (+) correlation [35]. In addition, Kim Bong-jung et al. (2019) examined the effect of hospital nurses' communication ability and nursing work environment on job satisfaction. The results of this study showed that job satisfaction had a significant correlation with communication ability [36]. Therefore, it is suggested that smooth communication among corporate members can have a positive effect on job satisfaction.

\subsection{Management trust related research}

Kim Se-won \& Baek Yoo-sung (2017) verified the mediating effect of trust in supervisors in the effect of emotional leadership on organizational commitment. As a result of the study, it was confirmed that selfawareness and relationship management ability among the components of emotional leadership had a significant positive $(+)$ effect on the trust of supervisors of organizational members, and supervisor trust had a positive effect on organizational commitment that can provide new job satisfaction [37]. Therefore, it can be estimated that management trust can have significant moderating effects on the impact of strategic leadership on job satisfaction. In addition, Yeo In-gil \& Kim Sung-soo(2005) analyzed the effect of leadership type on organizational performance according to the role of trust. As a result of the study, it was verified that trust relationship plays an important role in the relationship between branch manager and subordinate staff, and it was confirmed that leadership type and organizational performance have a moderating effect according to the trust of leader [38].Considering that organizational smooth communication is one of organizational performance, it is suggested that the moderating effect of managerial reliability can be significant in the effect of strategic leadership on organizational communication ability.

\section{Research design and methods}


This study is to examine the effect of strategic leadership on communication and organizational satisfaction in the organization and the moderating effect of management trust based on the previous studies. The shown in

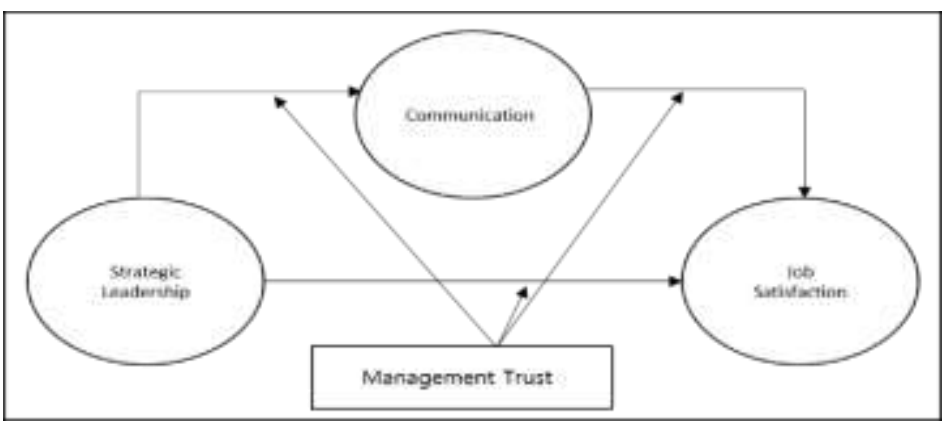
research model as Figure 1 is presented.

Figure 1 Research Model

3.1 Research hypothesis

In this study, the following hypotheses were set based on the contents of previous studies.

Hypothesis 1: Strategic leadership will have a positive $(+)$ effect on job satisfaction in an organization.

Hypothesis 2: Communication will have a positive (+) effect on job satisfaction.

Hypothesis 3. Strategic leadership will have a positive (+) effect on job satisfaction.

Hypothesis 4 . There will be a moderating effect on the trust of management between groups.

\subsection{Sample selection}

This study selected the members of the organization as the subjects of the study, surveyed them, and extracted 300 samples from the small and medium enterprises in Gyeonggi-do. 276 copies were used for analysis except for inappropriate questionnaires.

\subsection{Manipulating definition and measurement of variables}

Strategic leadership means that management presents vision, goals and delivers to organizational members, and six questions (goal presentation, convergence, and delivery) are referred to by Hambrick \& Mason (1984) in reference to the goal/vision strategic control core competencies and human resource development. operating development) etc.

Communication means communication channel, acceptance, and information sharing among organizational members. Based on the items presented by Baek Bo-kyung (2013), eight questions (communication channel, active acceptance, grievance treatment system, communication between the upper and lower levels, business cooperation, information sharing, and work process rationality) were composed.

Job satisfaction means the emotional state felt by the members of the organization during the work, and it consists of 5 questions (job satisfaction, promotion system fairness, competency difference, compensation system fairness, compensation level, and welfare system) with reference to the items presented by Bae Jae-hong (2008).

Management trust is a belief in management, which means that organizational members support management. The measurement tools are various, but this study used the organizational trust scale developed by cook and wall(1980). Cook and Wall's organizational trust scale consists of the sub-factors of management trust and member trust. The measurement questions about management trust were revised to three questions.

The questionnaire consisted of 22 items. For variable questionnaire items, one point was measured as 'very not' and five points were measured as 'very yes'. The summary result is the same as Table 1.

Table1 Composition of survey

\begin{tabular}{l|l|l}
\hline Measurement variables & Questions & Preceding studies \\
\hline Strategic Leadership & 6 & Hambrick \& Mason (1984) \\
\hline Communication & 8 & Baek Bo-kyung(2013) \\
\hline Job Satisfaction & 5 & Bae Jae-hong(2008)) \\
\hline
\end{tabular}




\begin{tabular}{l|l|l}
\hline Management Trust & 3 & Cook and wall(1980) \\
\hline Total & 22 & - \\
\hline
\end{tabular}

\subsection{Sample characteristic}

The general characteristics of the sample are equal to Table 2.The age group was 46 in 20s, 90 in 30s, 71 in 40s, 69 in 50s or older, and the number of years of service was 108 in less than 5 years, 58 in 5 to 9 years, and 110 in more than 10 years.

Table 2. The Characteristics of Samples

\begin{tabular}{l|l|l|l}
\hline \multicolumn{2}{l|}{ Categories } & \multicolumn{1}{l}{ Frequency } & \% \\
\hline \multirow{4}{*}{ Age } & $20 \mathrm{~s}$ & 46 & 16.7 \\
\cline { 2 - 4 } & $30 \mathrm{~s}$ & 90 & 32.6 \\
\cline { 2 - 4 } & $40 \mathrm{~s}$ & 71 & 25.7 \\
\cline { 2 - 4 } Service years & 50 and Up & 69 & 25.0 \\
\cline { 2 - 4 } & Total & 108 & 100 \\
\hline & Less than 5 years & 58 & 21.0 \\
\cline { 2 - 4 } & 5 to 9 years & 110 & 39.9 \\
\cline { 2 - 4 } & more than ten years & 276 & 100 \\
\cline { 2 - 4 } & Total & &
\end{tabular}

\subsection{Validity and reliability analysis}

The validity and reliability of the items were analyzed. In order to verify the validity, exploratory factor analysis was conducted, and main component analysis and orthogonal rotation method were adopted to simplify factor extraction and factor loading values. The eigenvalue was 1.0 or more and factor loading value was 0.4 or more.

In the factor analysis process, there was no removal, and the total variance explained was $81.56 \%$. The variables were extracted into three categories, and named as strategic leadership, job satisfaction, and communication. Because there is no factor that hinders the level of trust, all items are used for analysis, and the Cronbach' value of each variable is .939 like Table 3. It is distributed in 951, which is confirmed to be reliable.

Table. 3 Exploratory Factor Analysis and Reliability Test

\begin{tabular}{|c|c|c|c|c|}
\hline & SL & JS & CC & $\mathrm{C} \alpha$ \\
\hline SL3 & 882 & & & \\
\hline SL4 & 879 & & & \\
\hline SL6 & 874 & & & م2006 \\
\hline SL5 & 868 & & & ד \\
\hline SL2 & 721 & & & \\
\hline SL1 & 708 & & & \\
\hline JS3 & & 919 & & \\
\hline JS4 & & 906 & & \\
\hline JS1 & & 884 & & .951 \\
\hline JS2 & & 878 & & \\
\hline JS5 & & 856 & & \\
\hline CC4 & & & .926 & \\
\hline CC 3 & & & .901 & \\
\hline CC2 & & & .889 & .943 \\
\hline CC5 & & & .858 & \\
\hline CC1 & & & .787 & \\
\hline$\overline{\mathrm{EV}}$ & 4.46 & 4.37 & 4.22 & \\
\hline EV\% & 27.91 & 27.31 & 26.35 & \\
\hline CV\% & 27.91 & 55.22 & 81.56 & \\
\hline
\end{tabular}

\subsection{Measurement model analysis}


The hypothesis relationship between the constructs was analyzed by confirmatory factor analysis to verify the single dimension of each measurement variable prior to analysis. To verify the fit of the data, CMIN/DF(<3.0), GFICFINFIIFI $(>0.9)$, AGFI $(>0.8), \operatorname{RMR}(<0.05)$, and $\operatorname{RMSEA}(<1.0)$ were used. For this purpose, the removal process was repeated based on the squared multiple correlation value.

Finally, as a result of analyzing strategic leadership 1, 2, and 4 such as Table 4, job satisfaction 1, 2, and 5 after removing communication 1, 5, all the parameter estimates for the relationship between the latent variables and the measurement variables were 0 or more, and the $t$ value for these estimates exceeded 1.965 , and the squared multiple correlation value was 0.4 or more, indicating that the latent variables explain the variations of the measurement variables well. It was confirmed. In addition, the result of checking the value of the fit index showed that it was appropriate, and $t$ value was interpreted as significant when it was \pm 1.96 or more as the criterion for adopting or rejecting the hypothesis. The squared multiple correlation means the degree to which the variance of specific endogenous variables is explained by exogenous variables.

Table 4. Confirmatory Factor Analysis and Goodness of Fit of Measurement Model

\begin{tabular}{|c|c|c|c|c|c|c|c|c|}
\hline Measurement variables & & RW & SRW & S.E & C.R. & $\mathbf{P}$ & SMC & \\
\hline \multirow{2}{*}{ Sl } & 6 & 1.000 & 0.964 & - & + & - & 0.929 & \\
\hline & 5 & 0.900 & 0.855 & 0.077 & 11.672 & $* * *$ & 0.731 & \\
\hline \multirow{3}{*}{$\mathrm{Cc}$} & 2 & 0.938 & 0.906 & 0.04 & 23.196 & $* * *$ & 0.82 & \\
\hline & 3 & 1.064 & 0.929 & 0.044 & 24.39 & *** & 0.863 & \\
\hline & 4 & 1.000 & 0.904 & - & - & 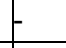 & 0.817 & \\
\hline \multirow{2}{*}{ JS } & 3 & 0.997 & 0.873 & 0.087 & 11.441 & $* * *$ & 0.762 & \\
\hline & 4 & 1.000 & 0.993 & - & $F$ & & 0.986 & \\
\hline \multirow{2}{*}{\multicolumn{3}{|c|}{ Goodness of fit - measurement model }} & \multicolumn{6}{|c|}{$\begin{array}{l}\mathrm{CMIN}=644.618, \quad \mathrm{DF}=101, \quad \mathrm{CMIN} / \mathrm{DF}=6.382, \quad \mathrm{GFI}=0.76, \\
\mathrm{CFI}=0.88, \mathrm{NFI}=0.86, \mathrm{IFI}=0.89, \mathrm{RMR}=0.056, \mathrm{RMSEA}=0.14\end{array}$} \\
\hline & & & \multicolumn{5}{|c|}{$\begin{array}{l}\text { GFI }=0.97, \quad \text { AGFI }=0.93, \quad \text { CFI }=0.98, \quad \text { NFI }=0.98, \quad I F I=0.98, \\
\text { RMSEA }=0.075\end{array}$} & $\mathrm{RMR}=0.024$, \\
\hline
\end{tabular}

\subsection{Structural model analysis}

To test the research model, the suitability of the formal model of the structural room was confirmed as the item of the final measurement model. The statistical research model was the same as Fig. 2, and the suitability result was the same as Table 5 .

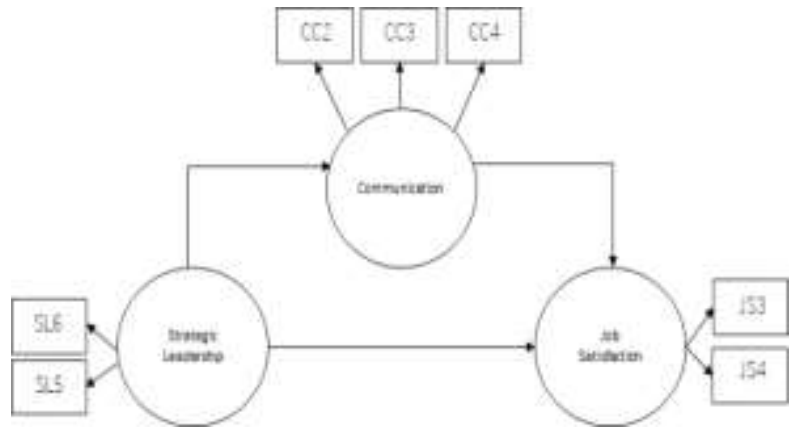

Figure 2 Statistical Research Model

Table 5. Goodness of Fit of Research Model

\begin{tabular}{l|l|l}
\hline & Reference Value & Measured Value \\
\hline Chi-Square & - & 27.815 \\
\hline $\mathrm{df}$ & - & 11 \\
\hline $\mathrm{P}$ & $>.05$ & .003 \\
\hline CMIN/DF & $<3$ & 2.529 \\
\hline GFI & $>.9$ & .973 \\
\hline AGFI & $>.8$ & .931 \\
\hline CFI & $>.9$ & .989 \\
\hline NFI & $>.9$ & .962 \\
\hline IFI & $>.9$ & .989 \\
\hline
\end{tabular}




\begin{tabular}{l|l|l}
\hline RMR & $<.05$ & .024 \\
\hline RMSEA & $<.1 .0$ & .075 \\
\hline
\end{tabular}

The research model was found to be suitable, and the path coefficients were examined for the specific hypothesis test, and the results were as follows: Table 6 and Fig. 3. The higher the perception of strategic leadership, the higher the perception of communication (standardization coefficient $=.383, p<.001$ ), and communication did not affect job satisfaction. The higher the perception of strategic leadership, the higher the perception of job satisfaction (standardization coefficient $=.319, \mathrm{p}<.001$ ). The size of the path can be directly compared with the standardization coefficient, and the larger the value, the greater the influence.

Table 6. The Structural Path Coefficients of the Research Model

\begin{tabular}{l|l|l|l|l|}
\hline Structural Path & P-value \\
\hline
\end{tabular}

Figure 3 The Structural Path

3.8 Control effect test

In order to determine whether the same analysis results can be obtained from two samples (involuntary application group and voluntary application group) extracted from the same population before confirming the path difference between the two groups, Multi-Sample Confirmatory Factor Analysis (MCFA) was conducted. The measurement identity was secured between the two samples because the fit of the measurement model did not deteriorate even after setting the regression coefficient together in the non-constrained model. The results are the same as Table 7.

Table 7. Multi-Sample Confirmatory Factor Analysis

\begin{tabular}{l|ll}
\hline Division & Unconstrained Model & Measurement Weights Model \\
\hline Chi-Square & 55.007 & 58.098 \\
\hline Chi-Square Difference & 3.091 & \\
\hline P-value of Chi-Square Difference & .543 & \\
\hline
\end{tabular}

The structural model analysis was conducted on the research model after dividing it into low-trust group and high-level group. The model was suitable for two groups, and the path coefficient for hypothesis testing was confirmed. The result is the same as Table 8.

Table 8. The Structural Path Coefficients of the Groups

\begin{tabular}{ll|l|l|l|l}
\hline \multirow{2}{*}{ Structure Path } & \multicolumn{4}{l}{ low group } & \multicolumn{1}{l}{ high group } \\
\cline { 3 - 6 } & & $\mathbf{B}(\mathbf{t})$ & $\mathbf{p}$ & $\mathbf{B}(\mathbf{t})$ & $\mathbf{p}$ \\
\hline SL $\quad$ CC & $176(1.755)$ & 0.079 & $.352(3.870)$ & $* * *$
\end{tabular}




\begin{tabular}{lllllll}
$\mathrm{CC}$ & $\rightarrow$ & $\mathrm{JS}$ & $-.013(-.197)$ & 0.844 & $.368(4.508)$ & *** \\
$\mathrm{SL}$ & $\rightarrow$ & JS & $.124(1.516)$ & 0.13 & $.428(4.956)$ & $* * *$ \\
\hline
\end{tabular}

The above results of the hypothesis test are the same as Table 9.

Table 9. The Results of Hypothesis Testing

\begin{tabular}{l|l|l}
\hline Hypothesis & Hypothesis to Be Tested & Result \\
\hline H1 & Strategic leadership will have a positive (+) effect on communication. & Accept \\
\hline H2 & Communication will have a positive (+) effect on job satisfaction. & Accept \\
\hline H3 & PStrategic leadership will have a positive $(+)$ effect on job satisfaction. & Accept \\
\hline H4 & There will be a difference in the moderating effect according to trus. & Accept \\
\hline
\end{tabular}

\section{Conclusion}

This study was conducted to identify the effects of strategic leadership on communication and job satisfaction in the organization and to identify the moderating effects of management trust in small and medium enterprises located in Gyeonggi-do. Based on the previous studies, the definition and dimension of each factor were identified, and the validity, reliability analysis, and measurement model of the measurement items were analyzed and used for the final analysis. The results of hypothesis verification of this study based on the structural equation model are as follows.

First, the perception of strategic leadership (vision/goal presentation, opinion gathering, flexibility management, and internal consensus formation efforts) had a positive effect on communication (channel, opinion gathering, complaint handling system, work cooperation and information sharing). Therefore, when the CEO of an organization presents vision and goals to the members according to strategic thinking in an uncertain market environment and achieves internal agreement by securing flexibility to collect their opinions, it is highly likely that the organization will develop more cohesively by coordinating work and common opinions according to smooth information sharing among the members.

Second, communication was found to be significant in job satisfaction (job satisfaction, sense of accomplishment, working environment, whether to leave). These results suggest that if communication between members is efficient and smooth, it can lead to ideal working environment, which leads to job satisfaction and achievement, and ultimately has a positive impact on organizational performance. Therefore, CEOs need to make effective efforts to cooperate with each other by securing channels for employees to communicate with each other by appropriately utilizing strategic leadership for promoting communication among the members and by achieving smooth information sharing between managers, members and members by listening to complaints.

Third, the perception of strategic leadership had a positive effect on job satisfaction. In other words, when the organization achieves internal consensus according to flexibility by presenting clear goals and visions in an uncertain market environment and fully reflecting the opinions of the employees in management, the achievement of the employees increases and the intention of turnover decreases. Therefore, CEOs need to develop a path to improve organizational performance by enhancing job satisfaction of employees by having strategic leadership to flexibly cope with the environment, which is more diversified than traditional and conservative leadership.

Fourth, there was a difference in the path between the groups on trust in management, and that group had a significant effect on all paths. However, in the case of high group, strategic leadership had a positive effect on communication and job satisfaction. Therefore, if management improves communication ability and job satisfaction of the organization members according to effective strategic leadership for restoring trust of the organization members and systematically and convincingly organizes the results of the leadership effect and feedbacks to the organization members, the reliability of the management of the organization members will rise and this trust will serve as a catalyst for improving their communication ability and job satisfaction.

The results of this study suggest that when CEOs use active and active uncertain information to connect with performance in today's rapidly changing market environment, they can improve communication skills among members and increase job satisfaction, which can lead to secondary performance by employees in addition to performance due to management power. In addition, when strategic leadership performance is effectively informed and accepted to employees, this effect can be confirmed. Therefore, CEOs should focus on forming organizational culture that can create trust in management, communication ability, and job satisfaction by actively completing continuous efforts to establish strategic leadership and education in related fields.

This study has the following limitations: First, it is desirable to analyze more companies in terms of small and medium enterprises. Second, if we analyze the industry and scale by subdividing it, we can 
draw more diverse and useful implications.

\section{Acknowledgment}

This research was financially supported by Hansung University.

\section{References}

[1] Yun-Sae Ra. (2017). The effect of strategic leadership of supervisor on creativity of member. Master`s thesis. Kwangwoon University.

[2] Lee-Hyun Joo. (2017). The Mediating Role of Transformational Leadership on Organizational Commitment of Teacher in the Alternative School: The Influence of Trust and Communication in the Organization. Doctor`s thesis. Sungkyunkwan University.

[3] Kim-Jang Hwan. (2013). The Effect of Communication in Organization Perceived by Social Welfare Officials on Job Satisfaction and Job Commitment: Mediating Effect of Interpersonal Relationship Ability. Master`s Degree. Hoseo University.

[4] Song-Ki Hyun. (2013). The Effect of Employee's Communication Competence and Communication Satisfaction on Job Satisfaction. Kangnam University.

[5] Kang-Hyun. (2017). The effects of strategic human resource management in industrial security on job attitudes and turnover intentions. Master`s Degree. Yongin University.

[6] Marshall, E. M. (2000). Building trust at the speed of change: The power of the relationship-based corporation (pp. 64-87). Toronto, ON: American Management Association.

[7] Yu. J. O., \& Choi. S. W. (2014). Exploring Strategic Leadership Competence of the Principals. The Korean Journal of the learning Sciences. 8(1):18-41.

[8] Kim. Y. C., \& Baek. Y. S. (2011). A Study on Components of Strategic Leadership - Senior Echelon of Military. Journal of Human Resource Management Research. 18(3). 219-241.

[9] Yoon, S. H. (2020). Analysis on Educational Needs for Development of Principals' Strategic Leadership. Journal of Practical Engineering Education, 12(1), 231-242.

[10] Kim, S. G. (2013). A Study on Relationship Among Strategic Leadership, Resistance and Perceived Performance After M\&A-Focused on IT Companies. Journal of the Korea Society of Computer and Information, 18(10), 183-192.

[11] Finkelstein, S., Hambrick, D., \& Cannella, A. A. (1996). Strategic leadership. St. Paul: West Educational Publishing.

[12] reland, R. D., \& Hitt, M. A. (1999). Achieving and maintaining strategic competitiveness in the 21st century: The role of strategic leadership. Academy of Management Perspectives, 13(1), 43-57.

[13] Rowe, W. G. (2001). Creating wealth in organizations: The role of strategic leadership. Academy of Management Perspectives, 15(1), 81-94.

[14] Hambrick, D. C., \& Mason, P. A. (1984). Upper echelons: The organization as a reflection of its top managers. Academy of management review, 9(2), 193-206.

[15] Bo-Kyung Baek. (2013). Effects of Office Worker's Communication and Communicative Competence on Burnout. Master's thesis, Myongji University.

[16] Min-Suk Nam. (2006). Elementary School Children's Perception of the Relationship between Father, Mother-Child Communication and School Adaptation. Master's thesis Chuncheon National University of Education.

[17] Beach, D. M., \& Reinhartz, J. (2000). Supervisory leadership: Focus on instruction. Allyn \& Bacon.

[18] Green, R. L. (2001). Practicing the art of leadership: A problem-based approach to implementing the ISLLC standards. Merrill.

[19] Hamilton, C., Parker, C., Smith, D. D., \& Rossi, M. J. (1983). Communicating for results: A guide for business and the professions. IEEE Transactions on Professional Communication, (2), 91-91.

[20] Reinhartz, J., \& Beach, D. M. (2004). Educational leadership: Changing schools, changing roles. Allyn \& Bacon.

[21] Jeong-Hoon Son. (2003). (An) empirical study on effects of communication ability on job satisfaction and organizational commitment. Master's thesis. Yeungnam University.

[22] Hoppock, R., Job Satisfaction, New York : Harper and Brothers Publishers, 1935

[23] Reitz, J. H. (1981). Behavior in Organizations. Homewood, Illinois: Richard D. Irwin. Inc. Robbins, Stephen P, 516.

[24] Locke, E. A. (1976). The nature and causes of job satisfaction. Handbook of industrial and organizational psychology. Chicago: RandMc Narlly

[25] Cook, J., \& Wall, T. (1980). New work attitude measures of trust, organizational commitment and personal need non-fulfilment. Journal of occupational psychology, 53(1), 39-52.

[26] Mayer, R. C., Davis, J. H., \& Schoorman, F. D. (1995). An integrative model of organizational trust. 
Academy of management review, 20(3), 709-734.

[27] Young-seok Lee (2004). A study on determinants and effectiveness of company trust and supervisor trust. A thesis on doctoral degree at Sungkyunkwan University.

[28] Fineman, S. (1993). Organizations as emotional arenas.

[29] Myung-eon Kim, \& Young-seok Lee. (2000). The Korean bases of workers` trust and distrust in their superiors Journal of Psychology: Cultural and Social Problems, 6(3), 99-120.

[30] Ju-il Lee. (2001). Emotion in the Workplace : Trust and Distrust between Leader and Members. Journal of Psychology: General, 20(1), 91-128.]

[31] Shin-S. I. (2018). Structural Relationships among Secondary School Principals' Compassionate Rationalism Leadership, Communication in Organization, Learning Organization Culture, Teaching Competency and Active Participation Degree in Teacher Learning Communities. The Journal of Korean Teacher Education. 35(4). 41-70.

[32] Kong, M., Park, J., Shin, Y., \& Sohn, Y. W. (2018). The Relationship between Empowering Leadership, Directive Leadership, Safety Communication, and Safety Behavior for Air Force Combat Pilots: The Moderated Mediating Effect of Safety Motivation. Journal of the Korean Society for Aviation and Aeronautics, 26(2), 8-30.

[33] Lee, Y. C. (2019). Effects of Transformational and Authentic Leadership on Crew Member's Job Satisfaction and Organizational Commitment: Mediating Effects of Crew Member's Self-efficacy and LMX. Journal of the Korean Society for Aviation and Aeronautics, 27(3), 54-69.

[34] Lee, K. K., \& Yi, S. G. (2015). Moderating Effects of Subordinates' Active Engagement between Commanders' Transformational- Transactional Leadership and Job Satisfaction. The Journal of the Korea Contents Association, 15(12), 447-458.

[35] Lee, Y. C. (2019). Effects of Transformational and Authentic Leadership on Crew Member's Job Satisfaction and Organizational Commitment: Mediating Effects of Crew Member's Self-efficacy and LMX. Journal of the Korean Society for Aviation and Aeronautics, 27(3), 54-69.

[36] Kim, B., Lee, S. Y., An, G. J., Lee, G., \& Yun, H. J. (2019). Influence of communication competency and nursing work environment on job satisfaction in hospital nurses. Journal of Health Informatics and Statistics, 44(2), 189-197.

[37] Kim. S. W., \& Baek. Y. S.(2017). The Effects of Emotional Leadership on Trust in Leader and Organizational Commitment. Ordo Economics Journal. 20(1). 91-109.

[38] Yu. I. K., \& Kim. S. S. (2005). The Effect of Leadership Type on Organizational Performance According to the Role of Trust. - Focused on Securities Companies-. Korean Journal of Business Administration. 18(2). 557-581. 